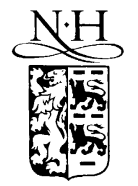

ELSEVIER

\title{
Kinetics of tertiarybutylphosphine adsorption and phosphorus desorption from indium phosphide $(001)$
}

\author{
Y. Sun, D.C. Law, S.B. Visbeck, R.F. Hicks * \\ Department of Chemical Engineering, University of California (UCLA), 405 Hilgard Avenue, Los Angeles, CA 90095-1592, USA
}

Received 18 October 2001; accepted for publication 17 April 2002

\begin{abstract}
The kinetics of tertiarybutylphosphine adsorption and phosphorus desorption from indium phosphide (001) have been determined using reflectance difference spectroscopy for real-time monitoring of the phosphorus coverage. The precursor adsorption rate depends linearly on the coverage, and the initial sticking coefficient varies from 0.007 to 0.001 as the temperature increases from 420 to $520{ }^{\circ} \mathrm{C}$. The phosphorus desorption rate is first order in the coverage and exhibits an activation energy and pre-exponential factor of $2.4 \pm 0.2 \mathrm{eV}$ and $10^{14.7 \pm 1.5} \mathrm{~s}^{-1}$. These reaction kinetics play an important role in the growth of phosphide-based alloys by metalorganic vapor-phase epitaxy. (c) 2002 Elsevier Science B.V. All rights reserved.
\end{abstract}

Keywords: Indium phosphide; Surface chemical reaction

\section{Introduction}

Metalorganic vapor-phase epitaxy (MOVPE) is widely employed in manufacturing compound semiconductor materials and devices. Many of the structures grown by MOVPE incorporate phosphide-based alloys, $\mathrm{L}_{x} \mathrm{M}_{1-x} \mathrm{~A}_{1-y} \mathrm{P}_{y}$, where $\mathrm{L}$ and $\mathrm{M}$ equal Al, Ga or In and A equals N, As or Sb [1-4]. These alloys have proven difficult to prepare due to the nonlinear dependence of the bulk composition on the partial pressure of the group $\mathrm{V}$ precursors [5,6]. The group $\mathrm{V}$ incorporation rate into the crystal lattice is not mass-transfer limited, but

\footnotetext{
${ }^{*}$ Corresponding author. Tel.: +1-310-206-6865; fax: +1-310206-4107.

E-mail address: rhicks@ucla.edu (R.F. Hicks).
}

instead is determined by the precursor adsorption and group $\mathrm{V}$ dimer desorption kinetics [7-12].

The rate of decomposition of tertiarybutylphosphine (TBP) has been examined in flow tubes by several authors $[13,14]$. In addition, the phosphorus desorption kinetics from indium phosphide (InP) and related materials have been studied in the MOVPE process using optical techniques [1517]. While these studies provide some qualitative insight into the reaction kinetics, the interpretation of these data is hindered by the fact that many different reactions occur simultaneously in these processes. For example, in an MOVPE reactor operating at 100 Torr, the phosphorus molecules desorbing from the surface slowly diffuse through a boundary layer and may undergo re-adsorption. Moreover, other species in the gas, such as $\mathrm{H}$ atoms and alkyl radicals can scavenge the group V elements from the surface. Therefore, it is not 
surprising that the rate parameters reported in the literature vary widely from one study to the next [15-17].

In this work, the kinetics of TBP adsorption and phosphorus desorption from $\operatorname{InP}(001)$ have been studied under well-controlled ultrahigh vacuum (UHV) conditions. Real-time monitoring of the phosphorus coverage at elevated temperatures during and after TBP dosing has been achieved using reflectance difference spectroscopy (RDS). Langmuir adsorption and first order desorption kinetics are observed. In addition, we discuss the mechanism of phosphorus incorporation and the implication of these results for the MOVPE of IIIV alloys.

\section{Experimental methods}

Indium phosphide films were grown in a horizontal MOVPE reactor at $530{ }^{\circ} \mathrm{C}$ with $6.5 \times 10^{-4}$ Torr of trimethylindium, $1.3 \times 10^{-2}$ Torr of TBP, and 20 Torr of hydrogen. The $\mathrm{H}_{2}$ carrier gas was passed through a SAES pure gas hydrogen purifier (PS4-MT3-H) to remove any remaining oxygen, nitrogen and carbon species. After growth, the samples were transferred directly to the UHV system. A J-Y NISEL RDS spectrometer was used to obtain the spectra and transient data [18].

Earlier studies by our group have shown that $\operatorname{InP}\left(\begin{array}{lll}0 & 0 & 1\end{array}\right)$ has three main surface structures, the $(2 \times 2),(2 \times 1)$ and $\delta(2 \times 4)$ phases with phosphorus coverages of $2.0,1.0$ and 0.125 , respectively $[19,20]$. The $(2 \times 2)$ exhibits two layers of phosphorus atoms, while the $(2 \times 1)$ is terminated with a single layer of dimerized phosphorus atoms. By contrast, the $\delta(2 \times 4)$ unit cell contains an InP dimer sitting on top of four indium dimers. Shown in Fig. 1 are the RD spectra for the $(2 \times 1)$ and $(2 \times 4)$ phases at room temperature and $500{ }^{\circ} \mathrm{C}$. The reflectance difference at $\sim 3.0 \mathrm{eV}$ has been shown to result from electronic transitions involving P-P dimers [21]. Inspection of the figure reveals that the $(2 \times 1)$ structure has a relatively intense positive peak at this energy, whereas the $(2 \times 4)$ exhibits negative anisotropy. Begarney et al. [22] have shown that the RD spectra of transitional structures at intermediate phosphorus

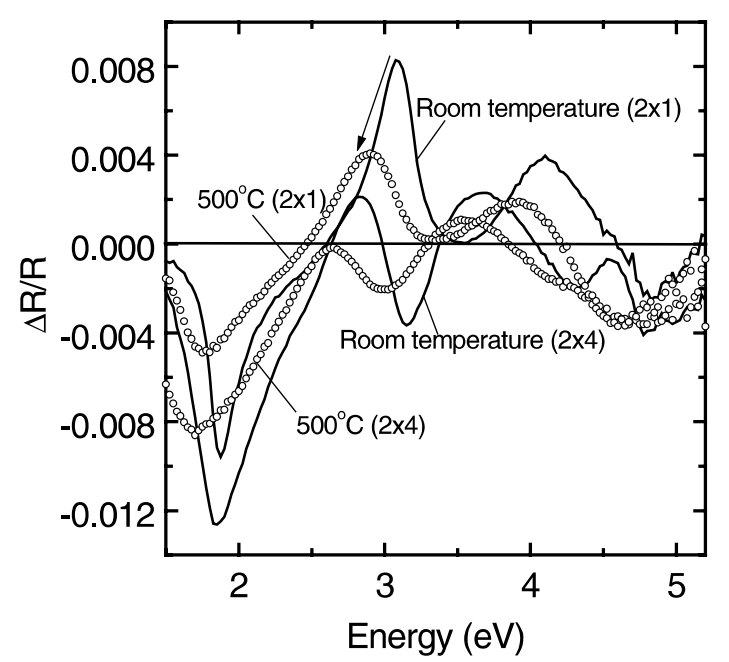

Fig. 1. Room temperature and $500{ }^{\circ} \mathrm{C}$ reflectance difference

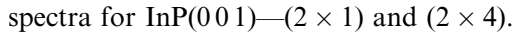

coverages are a linear combination of the pure phase spectra. We have used this concept to develop a method of monitoring the phosphorus coverage in real time, as described below.

As can be seen in Fig. 1, the P-dimer-related peak shifts to lower energy and broadens as the surface temperature rises. The peak position is a linear function of the surface temperature and has been calibrated in an earlier study by Visbeck et al. [23]. The temperature estimated from the peak position was found to be within $5^{\circ} \mathrm{C}$ of the temperature measured by a thermocouple attached to the sample holder.

The amount of phosphorus that may be desorbed from $\operatorname{InP}\left(\begin{array}{ll}0 & 0\end{array}\right)$ equals the $\mathrm{P}$ atoms on the $(2 \times 1)$ surface minus those on the $\delta(2 \times 4)$. Even though the $\delta(2 \times 4)$ contains some phosphorus in the top layer, these should not be counted as part of the $\mathrm{P}$ coverage in the kinetic studies, because this material only desorbs when the crystal undergoes congruent evaporation. Consequently, the following equation was used to estimate the phosphorus coverage:

$\theta_{\mathrm{P}}=\frac{I-I_{(2 \times 4)}}{I_{(2 \times 1)}-I_{(2 \times 4)}}$

In this equation, $I$ is the intensity of the Pdimer-related peak at an intermediate coverage, 
and $I_{(2 \times 1)}$ and $I_{(2 \times 4)}$ are the intensities of the Pdimer-related peak for the pure $(2 \times 1)$ and $(2 \times 4)$ phases, with all intensities measured at the same temperature. Isothermal adsorption data were recorded immediately after introducing TBP through a leak valve at pressures ranging from $10^{-7}$ to $10^{-4}$ Torr. The pressures were measured using an ion gauge filament and calibrated using a published correction factor [24]. Conversely, isothermal desorption data were taken immediately after closing the leak valve at background pressures below $10^{-9}$ Torr.

It should be noted that the $(2 \times 2) /(2 \times 1)$ reconstruction at a phosphorus coverage $>1.0$ cannot be distinguished from a pure $(2 \times 1)$ phase by RDS. Therefore, all the experiments were conducted at phosphorus coverages below 1.0.

\section{Results}

Fig. 2 shows a set of desorption curves taken at $465{ }^{\circ} \mathrm{C}$. The circles represent the experimental data with starting phosphorus coverages of $0.14,0.43$ and 0.90 , respectively. In these experiments, phosphorus dimers desorb from the surface as confirmed by the observation of $\mathrm{P}_{2}$ species in the gas by mass spectroscopy. The mass balance for the desorption process is

$\frac{\mathrm{d} \theta_{\mathrm{P}}}{\mathrm{d} t}=-R_{\mathrm{d}}$

In this equation, $\theta_{\mathrm{P}}$ is the time dependent phosphorus coverage and $R_{\mathrm{d}}$ the desorption rate in the units of $\mathrm{s}^{-1}$. For a first order reaction rate,

$R_{\mathrm{d}}=k_{\mathrm{d}} \theta_{\mathrm{P}}$

where $k_{\mathrm{d}}$ is the reaction rate constant. Combining Eqs. (2) and (3) and integrating both sides, we obtain

$\theta_{\mathrm{P}}=\theta_{\mathrm{P} 0} \exp \left(-k_{\mathrm{d}} t\right)$

where $\theta_{\mathrm{P} 0}$ is the initial phosphorus coverage when the TBP is turned off

The solid lines in Fig. 2 are the best fit of Eq. (4) to the experimental data. The desorption rate is first order in the phosphorus coverage and the $k_{\mathrm{d}}$ 's are found to be $0.028,0.024$ and $0.027 \mathrm{~s}^{-1}$. At each temperature, six to eight desorption experi-

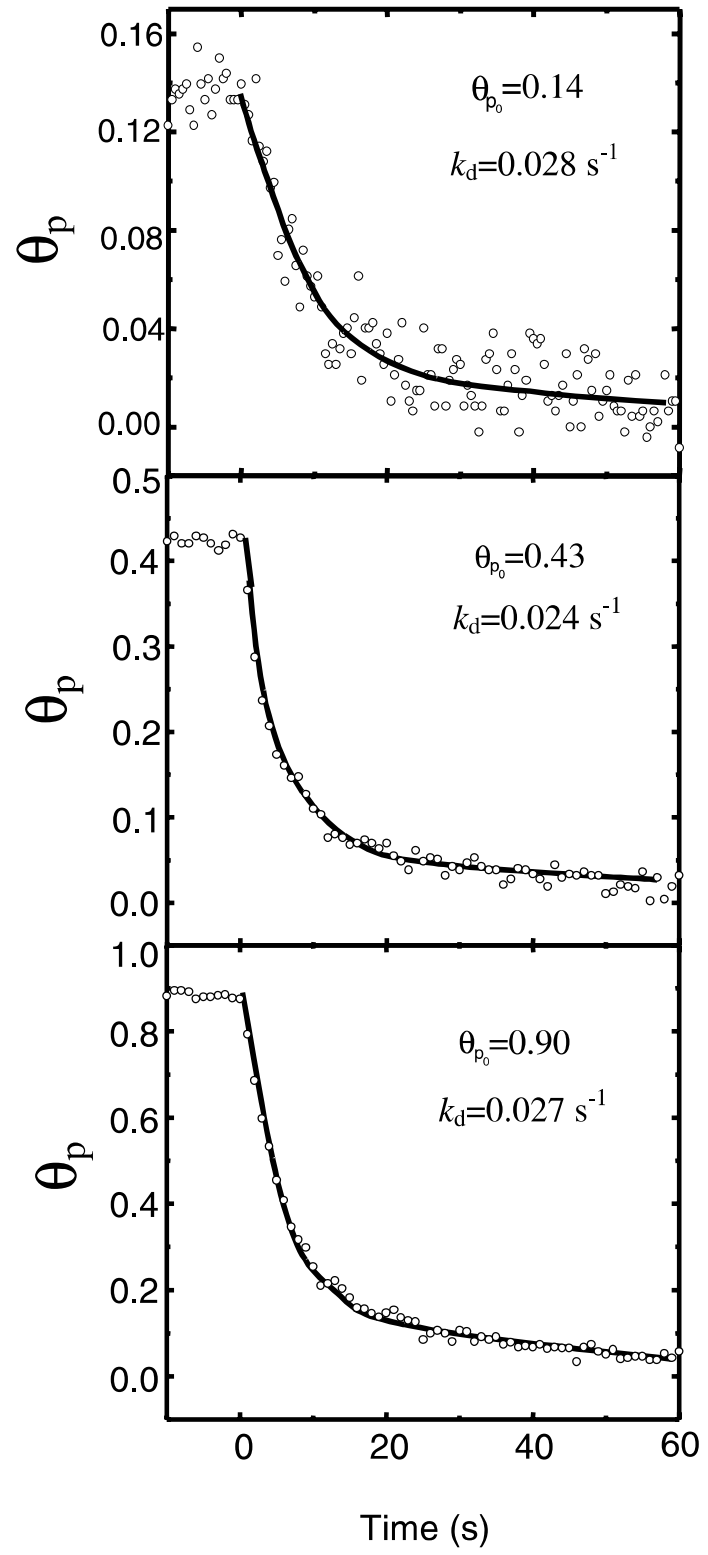

Fig. 2. Time-resolved desorption curves at $465^{\circ} \mathrm{C}$ and different initial coverages of phosphorus. Open circles are the experimental data and solid lines correspond to Eq. (4).

ments were performed and all the $k_{\mathrm{d}}$ 's averaged. The reaction rate constant at $465{ }^{\circ} \mathrm{C}$ is calculated to be $0.025 \pm 0.002 \mathrm{~s}^{-1}$. Since the desorption kinetics are independent of coverage, one may assume that lateral interactions between the adsorbates are negligible. 


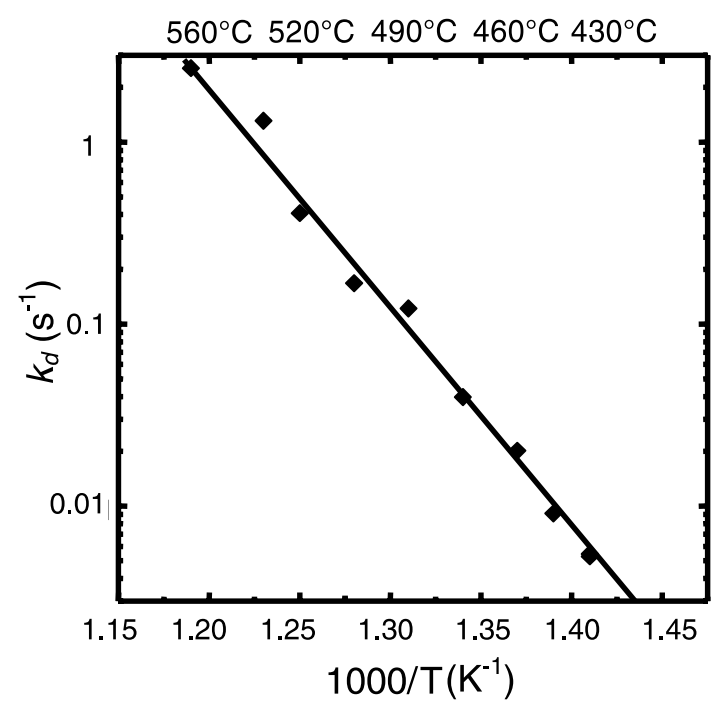

Fig. 3. Arrhenius plot of the phosphorus desorption rate constant, $k_{\mathrm{d}}$.

An Arrhenius plot is obtained when $k_{\mathrm{d}}$ is plotted in logarithm scale as a function of inverse temperature, as shown in Fig. 3. The activation energy and the pre-exponential factor of $k_{\mathrm{d}}$ are calculated to be $2.4 \pm 0.2 \mathrm{eV}$ and $10^{14.7 \pm 1.5} \mathrm{~s}^{-1}$, respectively. From transition-state theory, the preexponential factor has the form $(k T / h) \mathrm{e}^{\Delta S / k}$ with $\Delta S$ the entropy change in approaching the transition state, which is positive for desorption to the gas phase [25]. The exponential term contributes only one to two orders of magnitude and if $500{ }^{\circ} \mathrm{C}$ is used in the formula, a value of $10^{14}-10^{15}$ is obtained. Our experimental results are in good agreement with the theory.

We also recorded the time-resolved phosphorus coverage changes starting from a pure $(2 \times 4)$ phase, while dosing the surface at TBP pressures ranging from $10^{-7}$ to $10^{-4}$ Torr. Fig. 4 shows a set of TBP uptake curves at $465{ }^{\circ} \mathrm{C}$. The values indicated under each curve are the TBP dosing pressures. The behavior exhibited in the figure is indicative of Langmuir adsorption kinetics. In this case:

$R_{\mathrm{a}}=\frac{\frac{1}{4} v N_{\mathrm{TBP}} S_{0}}{N}\left(1-\theta_{\mathrm{P}}\right)$

where $R_{\mathrm{a}}$ is the TBP adsorption rate $\left(\mathrm{s}^{-1}\right), v$ is the mean molecular speed of TBP molecules $(\mathrm{m} / \mathrm{s}), S_{0}$

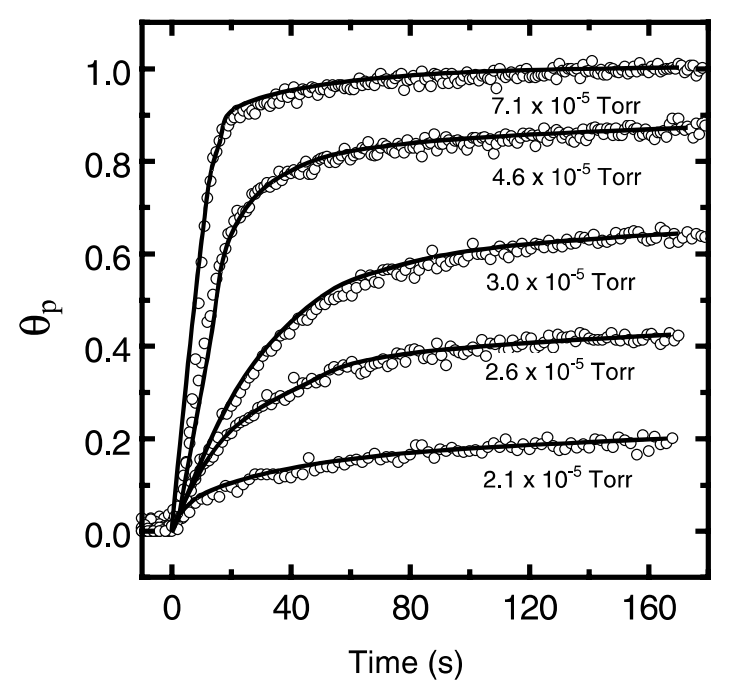

Fig. 4. Time-resolved up-take curves at $465{ }^{\circ} \mathrm{C}$ and different TBP pressures. Open circles are the experimental data and solid lines correspond to Eq. (7).

is the initial sticking coefficient, $N_{\mathrm{TBP}}$ is the number concentration of precursor molecules in the gas $\left(\mathrm{m}^{-3}\right)$, and $N$ is the site concentration $\left(5.8 \times 10^{18}\right.$ $\left.\left(\mathrm{m}^{-2}\right)\right)$ on the $\operatorname{InP}\left(\begin{array}{lll}0 & 0 & 1\end{array}\right)$ surface.

The mass balance on the adsorbed phosphorus during TBP dosing is given by

$\frac{\mathrm{d} \theta_{\mathrm{P}}}{\mathrm{d} t}=R_{\mathrm{a}}-R_{\mathrm{d}}=\frac{\frac{1}{4} v N_{\mathrm{TBP}} S_{0}}{N}\left(1-\theta_{\mathrm{P}}\right)-k_{\mathrm{d}} \theta_{\mathrm{P}}$.

Integrating Eq. (6) yields the following function for the phosphorus coverage:

$\theta_{\mathrm{P}}=\frac{k_{\mathrm{s}}}{k_{\mathrm{s}}+k_{\mathrm{d}}}\left(1-\exp \left(-k_{\mathrm{s}}+k_{\mathrm{d}}\right) t\right)$

with $\quad k_{\mathrm{s}}=\frac{\frac{1}{4} v N_{\mathrm{TBP}} S_{0}}{N}$.

In Fig. 4, the solid lines are the best fit of Eq. (7) to the experimental data. From the fit one obtains $k_{\mathrm{s}}$ and in turn $S_{0}$. At each temperature, six to eight experiments were performed with different precursor pressures, and the $S_{0}$ 's obtained from each curve were averaged. The initial sticking coefficient at $465{ }^{\circ} \mathrm{C}$ is $0.0044 \pm 0.0003$.

In Fig. 5, $S_{0}$ is plotted in logarithm scale as a function of inverse temperature, and a straight line is obtained. From the slope and intercept, 


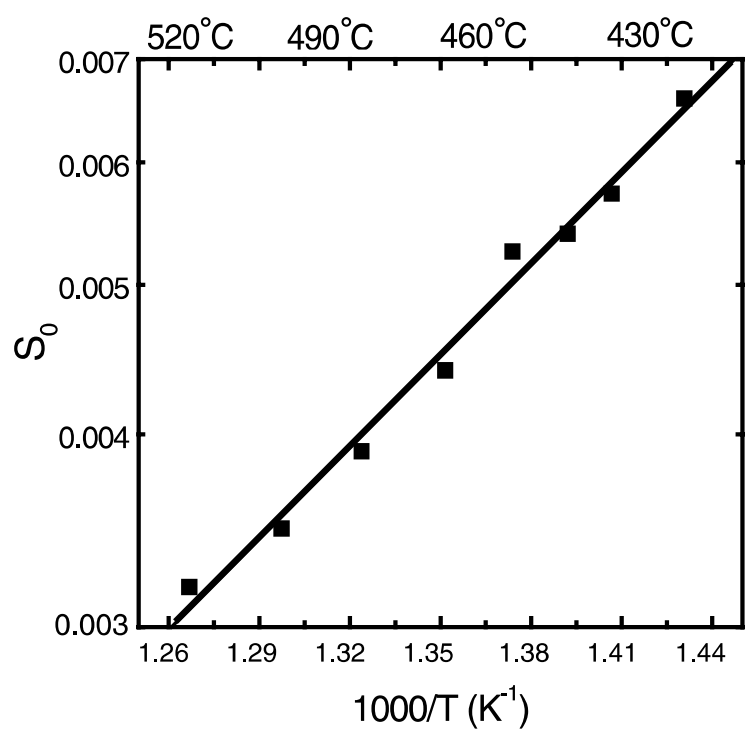

Fig. 5. Temperature dependence of the initial sticking coefficient of TBP.

the initial sticking coefficient equals $10^{-5.0 \pm 0.4} \times$ $\exp (0.39( \pm 0.04) \mathrm{eV} / k T)$. Note that the apparent energy barrier for TBP adsorption is $-0.39 \mathrm{eV}$, which results in $S_{0}$ decreasing with increasing temperature.

\section{Discussion}

We have found that the phosphorus desorption rate is first order in the phosphorus coverage, which is consistent with previous work [15-17]. Nevertheless, the values for the kinetic constants measured in previous studies do not agree with each other, or with the results presented here. In Table 1, the desorption rate kinetics are compared between the different studies. One sees that at 500
${ }^{\circ} \mathrm{C}$, the reported rate constant varies from 0.13 to $7.8 \mathrm{~s}^{-1}$. In the following paragraphs, several possible reasons for this discrepancy are proposed.

The earlier studies were performed in an MOVPE reactor with a constant $\mathrm{H}_{2}$ flow, resulting in a thin concentration boundary layer on top of the sample surface. Each research group used a specific total pressure and $\mathrm{H}_{2}$ flow rate, so that the boundary layer thickness was different in each case. This difference probably influenced their recorded desorption rates. For example, Féron et al. [17] found that when the total pressure in the reactor changed from 10 to 5 to $20 \mathrm{kPa}$, the desorption rate first increased then decreased. Another phenomenon that can occur in an MOVPE reactor is the desorption of group $\mathrm{V}$ elements from the substrate holder. This will result in lower apparent desorption rates. In our work, the TBP was turned off during desorption, yielding a background pressure of $10^{-9}$ Torr. Therefore, no boun- dary layer was present and no gas-phase processes affected the desorption rate.

Another important factor that may influence the rate data is the coverage range used during the desorption measurements. In this work, the phosphorus coverage remained below 1.0 (see Section 2 ), whereas in the earlier studies, the initial $P$ coverage was above 1.0. We know that if the $\operatorname{InP}\left(\begin{array}{lll}0 & 0 & 1\end{array}\right)$ surface is exposed to $10^{-2}$ Torr of TBP close to the growth temperature, the surface will become $(2 \times 2)$ reconstructed with a phosphorus coverage of about 1.5 ML [26]. Unfortunately, the top layer phosphorus islands are disordered so that the RDS line shape is identical to that of a clean $(2 \times 1)$. Nonetheless, the extra $\mathrm{P}$ dimers affect the desorption rates $[15,17]$. We find that when the surface is saturated with a higher TBP pressure than is necessary to maintain a pure $(2 \times 1)$ phase,

Table 1

Comparison of the phosphorus desorption rates obtained by different research groups

\begin{tabular}{lllll}
\hline References & Experimental conditions & \multicolumn{2}{l}{ Kinetics } & \\
\cline { 3 - 5 } & & $k_{\mathrm{d} 0}\left(\mathrm{~s}^{-1}\right)$ & $E_{\mathrm{d}}(\mathrm{eV})$ & $k_{\mathrm{d}}$ at $500{ }^{\circ} \mathrm{C}\left(\mathrm{s}^{-1}\right)$ \\
\hline Lee et al. [15] & MOVPE growth at 76 Torr & - & 3.36 & 0.30 \\
Kobayashi and Kobayashi [16] & MOVPE growth at 45 Torr & $10^{20.1}$ & 2.96 & 7.97 \\
Féron et al. [17] & MOVPE growth at 75 Torr & $10^{3.0}$ & 0.60 & 0.13 \\
This work & Surface sciences at $<10^{-9}$ Torr & $10^{14.7}$ & 2.4 & 0.16 \\
\hline
\end{tabular}


the apparent desorption rate is reduced $\left(k_{\mathrm{d}}\right.$ is roughly half that when the $\mathrm{P}$ coverage is below 1.0). Evidently, the extra phosphorus adsorbates serve as a reservoir to replenish the $(2 \times 1)$ structure, so that one observes a more gradual change in the RDS signal.

In this study, all the reaction experiments were performed under well controlled conditions that did not suffer from the complications that can occur in an MOVPE reactor. Moreover, the optical signal used for in situ monitoring of the phosphorus coverage was independently calibrated by scanning tunneling microscopy [22]. Therefore, we concluded that the equation, $k_{\mathrm{d}}=10^{14.7} \times$ $\exp (-2.4 \mathrm{eV} / k T)$ accurately represents the rate constant for $\mathrm{P}_{2}$ desorption from $\operatorname{InP}\left(\begin{array}{ll}0 & 0\end{array}\right)$.

We have found that the initial sticking coefficient of TBP on $\operatorname{InP}\left(\begin{array}{lll}0 & 0 & 1\end{array}\right)$ decreases with temperature, exhibiting an apparent energy barrier of $-0.39 \mathrm{eV}$. These results can be understood by considering the mechanism for the reactive adsorption of TBP. It combines the overall effects of adsorption, desorption and decomposition of the TBP molecules on the surface as illustrated by the following equations:

$*_{\text {In }}+$ TBP $(\mathrm{g}) \longleftrightarrow *_{\text {In }}$ TBP

$*_{\mathrm{P}}+*_{\text {In }} \mathrm{TBP} \longrightarrow *_{\mathrm{P}} \mathrm{L}+*_{{ }_{\mathrm{In}}} \mathrm{PL}_{2}$

$*_{\text {In }} \mathrm{PL}_{2} \longrightarrow *_{\mathrm{P}}+$ products

In Eq. (9), TBP undergoes reversible adsorption and desorption. This step is assumed to be in equilibrium. Elevating the surface temperature enhances the rate of molecular desorption more than that of adsorption, since the forward step is exothermic. Therefore, the concentration of $*_{\text {In }}$ TBP will be less as the temperature goes up. In Eq. (10), irreversible dissociation of the adsorbed TBP molecules occurs. Here, L is an alkyl group or a hydrogen atom. According to previous work by Fu et al. [27], this reaction is most likely the ratecontrolling step in the heterogeneous decomposition of the group V precursor. Lastly, reaction (11) is the sum of several fast elementary steps leading to the formation of phosphorus dimers.

Due to the competing temperature effect of reactions (9) and (10), $S_{0}$ will be of the form

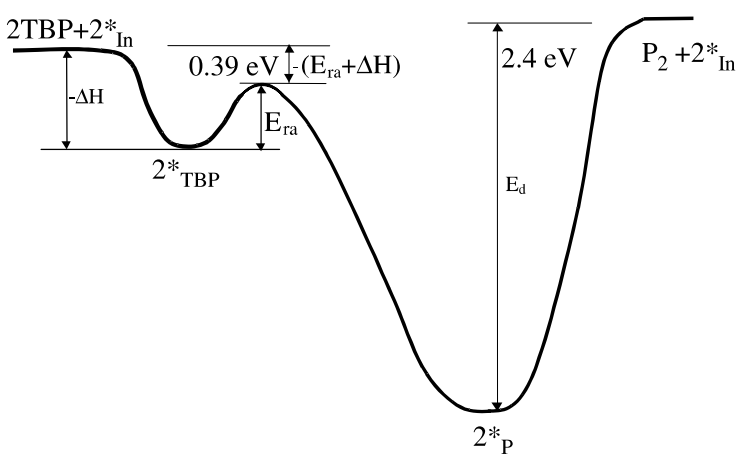

Fig. 6. Reaction pathway for TBP adsorption and phosphorus desorption.

$A \exp \left(-\left(E_{\mathrm{ra}}+\Delta H\right) / k T\right)$, where $E_{\mathrm{ra}}$ is the energy barrier for TBP dissociation, and $\Delta H$ is the heat of molecular adsorption. According to our transient adsorption measurements, the pre-exponential factor, $A$, equals $1.0 \times 10^{-5}$ and the energy barrier, $\left(E_{\mathrm{ra}}+\Delta H\right)$, equals $-0.39 \mathrm{eV}$.

Fig. 6 summarizes the TBP adsorption and phosphorus desorption processes using a potential energy diagram. One important conclusion is that if $\left(E_{\mathrm{ra}}+\Delta H\right)>0, S_{0}$ increases with temperature; whereas if $\left(E_{\mathrm{ra}}+\Delta H\right)<0, S_{0}$ decreases with temperature. The latter case holds here for TBP adsorption on $\operatorname{InP}\left(\begin{array}{ll}0 & 0\end{array}\right)$. The first case has been observed for $\mathrm{AsH}_{3}$ adsorption on $\mathrm{GaAs}\left(\begin{array}{lll}0 & 0 & 1\end{array}\right)$ using infrared spectroscopy [27]. Other phosphorus precursors, such as $\mathrm{PH}_{3}$, may exhibit either behavior.

One important application for the kinetic study presented here is controlling the composition of $\mathrm{In}_{x} \mathrm{Ga}_{1-x} \mathrm{As}_{y} \mathrm{P}_{1-y}$ alloys during MOVPE. These semiconductor alloys are often employed in photonic devices [28-32]. They are challenging to grow, because the incorporation of the group $\mathrm{V}$ elements in the film is governed by the reaction kinetics, yielding a nonlinear dependence of the group V superlattice composition on the feed rates of the arsenic and phosphorus precursors. Consequently, small changes in the amount of arsine or tertiarybutylarsine fed to the reactor lead to large changes in the atomic fraction of arsenic $(y)$ in the film. There have been several models in the literature attempting to explain this effect [7-12]. Although a lot of discrepancies in these models still exist, phosphorus desorption and precursor 
adsorption kinetics are crucial to predict the film composition. From the work presented here, we show that the surface reaction rates depend upon the substrate temperature, the precursor partial pressure, and the choice of the precursor molecule, e.g., TBP versus phosphine. Crystal growers should consider these issues when attempting to gain better control of their alloy growth processes.

\section{Acknowledgements}

Funding for this research was provided by the National Science Foundation, Divisions of Chemical and Transport Systems and Materials Research, and by the California Energy Innovations Small Grant Program.

\section{References}

[1] M. Meyer, Compd. Semicond. 3 (1997) 30.

[2] K.A. Jackson, Compound Semiconductor Devices: Structures and Processing, Wiley-VCH, Weinheim, Germany, 1998.

[3] M. Sugo, H. Mori, Y. Sakai, Y. Itoh, Appl. Phys. Lett. 60 (1992) 472

[4] K. Mobarhan, C. Jelen, E. Kolev, M. Razeghi, J. Appl. Phys. 74 (1993) 743.

[5] M.L. Hitchman, K.F. Jensen, Chemical Vapor Deposition: Principles and Applications, Academic, London, 1993.

[6] I. Kim, K. Uppal, W.-J. Choi, P.D. Dapkus, J. Cryst. Growth 193 (1998) 293.

[7] L. Samuelson, P. Omling, H.G. Grimmeiss, J. Cryst. Growth 61 (1983) 425.

[8] J.E. Cunningham, M.D. Williams, R.N. Pathak, W. Jan, J. Cryst. Growth 150 (1995) 492.

[9] B.W. Liang, C.W. Tu, J. Appl. Phys. 74 (1993) 255.

[10] E.T.J.M. Smeets, J. Cryst. Growth 82 (1987) 385.

[11] C.W. Tu, B.W. Liang, H.Q. Hou, J. Cryst. Growth 127 (1993) 251.
[12] N. Gopalakrishnan, R. Dhanasekaran, J. Cryst. Growth 162 (1996) 113.

[13] G.B. Stringfellow, Organometallic Vapor-Phase Epitaxy: Theory and Practice, Academic press, San Diego, 1989.

[14] C.W. Hill, G.B. Stringfellow, L.P. Sadwick, J. Cryst. Growth 181 (1997) 321.

[15] T.W. Lee, H. Hwang, Y. Moon, E. Yoon, J. Vac. Sci. Technol. A 17 (1999) 2663.

[16] N. Kobayashi, Y. Kobayashi, Jpn. J. Appl. Phys. Part 230 (1991) L1699.

[17] O. Féron, M. Sugiyama, W. Asawamethapant, N. Futakuchi, Y. Feurprier, Y. Nakano, Y. Shimogaki, Appl. Surf. Sci. 157 (2000) 318.

[18] M.J. Begarney, L. Li, C.H. Li, C.D. Law, Q. Fu, R.F. Hicks, Phys. Rev. B 62 (2000) 8092.

[19] L. Li, B.-K. Han, Q. Fu, R.F. Hicks, Phys. Rev. Lett. 82 (1998) 1879.

[20] L. Li, Q. Fu, C.H. Li, B.-K. Han, R.F. Hicks, Phys. Rev. B 61 (2000) 10223.

[21] D. Law, Q. Fu, S. Visbeck, Y. Sun, C.H. Li, R.F. Hicks, Surf. Sci. 496 (2001) 121.

[22] M.J. Begarney, C.H. Li, D.C. Law, S.B. Visbeck, Y. Sun, R.F. Hicks, Appl. Phys. Lett. 78 (2001) 55.

[23] S. Visbeck, T. Hannappel, M. Zorn, J.-T. Zettler, F. Willig, Phys. Rev. B 63 (2001) 245.

[24] R.L. Summers, Lewis Research Center, Cleveland, Ohio, NASA TN D-5285.

[25] R.I. Masel, Principles of adsorption and reaction on solid surfaces, Wiley, New York, 1996.

[26] L. Li, B.-K. Han, D. Law, C.H. Li, Q. Fu, R.F. Hicks, Appl. Phys. Lett. 75 (1999) 683.

[27] Q. Fu, L. Li, C.H. Li, M.J. Begarney, D.C. Law, R.F. Hicks, J. Phys. Chem. B 104 (2000) 5595.

[28] G. Rondeau, S. Biblemont, J. Decobert, G. Post, International Conference on Indium Phosphide and Related Materials, IEEE, Williamsburg, VA, USA, 2000, p. 333.

[29] D. Yap, K.R. Elliott, Y.K. Brown, A.R. Kost, E.S. Ponti, IEEE Photonics Technol. Lett. 13 (2001) 626.

[30] A. Huelsman, Compd. Semicond. (2001) 7.

[31] H. Takeuchi, K. Tsuzuki, K. Sato, M. Yamamoto, Y. Itaya, A. Sano, M. Yoneyama, T. Otsuji, IEEE J. Sel. Top. Quant. 3 (1997) 336.

[32] D. Huber et al., J. Lightwave Technol. 18 (2000) 992. 